\title{
The E2F-Regulated Gene Chk1 Is Highly Expressed in Triple-Negative Estrogen Receptor-/Progesterone Receptor-/HER-2 - Breast Carcinomas
}

\author{
Lieve Verlinden, ${ }^{1}$ Isabelle Vanden Bempt, ${ }^{3}$ Guy Eelen, ${ }^{1}$ Maria Drijkoningen, ${ }^{3,4}$ Ilse Verlinden, ${ }^{6}$ \\ Kathleen Marchal, ${ }^{2}$ Christiane De Wolf-Peeters, ${ }^{3}$ Marie-Rose Christiaens, ${ }^{4,5}$ Luc Michiels, ${ }^{6}$ \\ Roger Bouillon, ${ }^{1}$ and Annemieke Verstuyf ${ }^{1}$
}

${ }^{1}$ Laboratorium voor Experimentele Geneeskunde en Endocrinologie and ${ }^{2} \mathrm{CMPG} / \mathrm{ESAT}$, Katholieke Universiteit Leuven; ${ }^{3}$ Department of Pathology, ${ }^{4}$ Multidisciplinary Breast Centre, and ${ }^{5}$ Department of Surgery, University Hospital of the Katholieke Universiteit Leuven, Leuven, Belgium; and ${ }^{6}$ Biomedical Research Institute BIOMED, Universiteit Hasselt, Diepenbeek, Belgium

\begin{abstract}
We previously showed that checkpoint kinase 1 (ChkI) and Claspin, two DNA-damage checkpoint proteins, were downregulated by 1,25-dihydroxyvitamin $D_{3}$, a known inhibitor of cell proliferation. In the present study, we aimed to investigate the transcriptional regulation of Chkl and Claspin and to study their expression levels in human breast cancer tissue. Transient transfection experiments in MCF-7 breast cancer cells showed that promoter activities of ChkI and Claspin were regulated by the $\mathrm{E} 2 \mathrm{~F}$ family of transcription factors. Subsequently, transcript levels of Chkl, Claspin, and E2F1 were determined by quantitative reverse transcriptase-PCR analysis in 103 primary invasive breast carcinomas and were compared with several clinicopathologic variables in breast cancer. A strong correlation was found between ChkI and Claspin transcript levels. Transcript levels of Chk1, Claspin, and E2F1 were highest in histologic grade 3 tumors and in tumors in which the expression of estrogen receptor (ER) and progesterone receptor (PR) was lost. Moreover, Chk1 expression was significantly elevated in grade 3 breast carcinomas showing a triple-negative $\mathrm{ER}-/ \mathrm{PR}-/ \mathrm{HER}-2$ - phenotype compared with other grade 3 tumors. Further research is warranted to validate the use of Chk1 inhibitors in triplenegative breast carcinomas for which treatment strategies are limited at present. [Cancer Res 2007;67(14):6574-81]
\end{abstract}

\section{Introduction}

Eukaryotic cells have developed complex checkpoint pathways that monitor DNA for damage or incomplete replication. Checkpoint pathways are amplified upon detection of aberrant DNA structures and lead to a delay in cell cycle progression during which damage can be repaired or replication be completed. Alternatively, in case of heavily damaged or seriously deregulated cells, checkpoint activation can result in apoptosis. As such, checkpoint mechanisms are essential for the maintenance of genomic integrity (1). When vertebrate cells experience replication

Note: Supplementary data for this article are available at Cancer Research Online (http://cancerres.aacrjournals.org/).

L. Verlinden and I. Vanden Bempt contributed equally to this work.

Requests for reprints: Annemieke Verstuyf, Laboratorium voor Experimentele Geneeskunde en Endocrinologie, Katholieke Universiteit Leuven, Legendo, bus 902, Gasthuisberg, Herestraat 49, 3000 Leuven, Belgium. Phone: 32-16-346163; E-mail: Mieke.Verstuyf@med.kuleuven.be.

(C)2007 American Association for Cancer Research.

doi:10.1158/0008-5472.CAN-06-3545 arrest or undergo DNA damage by UV irradiation, the ATR kinase [ataxia telangiectasia mutated (ATM)- and Rad3-related kinase] phosphorylates and activates the Chk1 protein kinase. Activated Chk1 inhibits Cdc25 phosphatases, which control inhibitory phosphorylation sites on cyclin-dependent kinases, the latter being critical regulators of cell cycle transitions (2). Full activation of Chkl by ATR requires Claspin, which may act as a scaffolding protein that brings together ATR and Chk1 (3). Apart from being a signal mediator in checkpoint control, Claspin was suggested to act as a sensor of DNA replication (4). Moreover, Claspin is shown to be involved in cell proliferation as overproduction of Claspin stimulates cell growth, suggesting an oncogenic role for Claspin (5).

The common occurrence of checkpoint defects distinguishes cancer cells from normal cells, thereby providing a potential target for therapeutic intervention. As a possible strategy, checkpoint signaling can be inhibited to enhance the effect of concomitant treatment with DNA-damaging drugs or radiation. Targeting of ATM/ATR as well as Chk1 has already provided encouraging results $(1,6-8)$. Indeed, when the $G_{2}$ or $S$ checkpoint is abrogated by inhibition of Chkl, checkpoint-deficient cancer cells will undergo mitotic catastrophe and eventually apoptosis. However, also, Claspin may be a candidate to target as depletion of Claspin by small interference RNA increases the sensitivity of cultured human cells to genotoxic stress and promotes cell death (9).

We previously identified $C h k 1$ and Claspin in a cluster of genes that were quickly down-regulated in mouse MC3T3-E1 and in mouse mammary carcinoma cells by treatment with 1,25-dihydroxyvitamin $D_{3}$, which is a known inhibitor of cell proliferation (10). Approximately $30 \%$ of the genes in this down-regulated cluster were known to be transcriptional targets of the E2F family of transcription factors. In silico promoter analysis further showed an additional $20 \%$ of genes to contain E2F-binding sites in their promoter. Claspin was found to be present among those newly identified E2F target genes.

In the present study, we aimed to investigate Chk1 and Claspin expression in human breast cancer tissue. Because promoter analysis of human Claspin (during the course of our study; ref. 11) and Chkl (12) indicated that these genes were transcriptional targets of the E2F family of transcription factors, we sought to correlate Chk1 and Claspin transcript levels with those of E2F1. Chk1, Claspin, and E2F1 transcript levels were further compared with those of the cell proliferation marker Ki-67 and with several clinicopathologic variables in breast cancer such as tumor size and grade, estrogen receptor (ER) and progesterone receptor (PR) expression, HER-2 status, and the presence of lymph node metastases. 


\section{Materials and Methods}

Cell culture-proliferation assay and RNA extraction. MC3T3-E1 cells (Riken Cell Bank) were maintained in $\alpha$-MEM with $2 \mathrm{mmol} / \mathrm{L}$ glutaMAX-I containing 10\% heat-inactivated fetal bovine serum (FBS, Biochrom KG), $100 \mathrm{units} / \mathrm{mL}$ penicillin, and $100 \mu \mathrm{g} / \mathrm{mL}$ streptomycin (Invitrogen). MCF-7 cells (American Type Culture Collection) were maintained in DMEM with $2 \mathrm{mmol} / \mathrm{L}$ glutaMAX-I containing $10 \%$ heat-inactivated FBS, 100 units $/ \mathrm{mL}$ penicillin, and $100 \mu \mathrm{g} / \mathrm{mL}$ streptomycin. For serum starvation experiments, MC3T3-E1 cells were seeded in $\alpha$-MEM without FBS either in $25-\mathrm{cm}^{2}$ flasks (200,000 per flask) for cell cycle analysis or in six-well dishes $(65,000$ per well) for RNA extraction. After $48 \mathrm{~h}$, culture medium was replaced by medium with $10 \%$ FBS. For analysis of cell cycle distribution, cells $\left(10^{6}\right)$ were fixed in icecold $75 \%$ ethanol. Fixed cells were washed with PBS supplemented with $0.05 \%$ Tween 20 and resuspended in PBS with $0.5 \mathrm{mg} / \mathrm{mL}$ propidium iodide and $1 \mathrm{mg} / \mathrm{mL}$ RNase A (Sigma). Samples were analyzed with a FACSort flow cytometer (Becton Dickinson, BD Biosciences). Total RNA was isolated using the RNeasy kit (Qiagen).

Plasmids. Promoter fragments of murine Chkl (-223/+43), human Chkl $(-572 /+34)$, murine Claspin $(-250 /+70)$, and human Claspin $(-246 /+52)$ were obtained through PCR amplification and cloned into the pGL3-Basic reporter vector (Promega). Site-directed mutagenesis to mutate the E2Fbinding sites in the promoter regions of murine Chkl and Claspin was done by use of the QuickChange II Site-Directed Mutagenesis kit (Stratagene) according to the instructions of the manufacturer. For murine Chkl, the E2F-binding site TTTGGCGGGAAA (at position +9 to +20 relative to transcription start site) was mutated to TGTGACGTGACA. For murine Claspin, the E2F-binding site GCGCGAAA (at position -24 to position - 16 relative to transcription start site) was mutated to GCGTGACA. Expression plasmids pcDNA-HA-E2F1, E2F2, E2F3, and E2F4 were kind gifts of Dr. J. Nevins (Duke University Medical Center, Durham, NC). The CMV-HAE2F5 expression plasmid was a gift of Dr. J. Magae (Institute of Research and Innovation, Chiba, Japan). Mutant E2F1 (mutated at position 132) was a gift of Dr. K Helin (Copenhagen, Denmark). The $\beta$-galactosidase expression vector pcDNA3.1(-)/Myc-His/lacZ and the pcDNA3.1/Myc-His vector were obtained from Invitrogen.

Transfection assays. Exponentially growing MC3T3-E1 cells were transfected with Fugene 6 (Roche Diagnostics) in 24-well dishes $(20,000$ per well) with $100 \mathrm{ng}$ of luciferase reporter vector (or representative control vector), $50 \mathrm{ng}$ of the different E2F constructs (or the empty pcDNA3.1/MycHis), and $10 \mathrm{ng}$ of pcDNA3.1(-)/Myc-His/lacZ. Exponentially growing MCF-
7 cells were seeded in 24-well dishes (50,000 per well) and transfected with Fugene 6 with $500 \mathrm{ng}$ of luciferase reporter (or representative control vector), $250 \mathrm{ng}$ of the different E2F constructs (or empty pcDNA3.1/MycHis), and $25 \mathrm{ng}$ of pcDNA3.1(-)/Myc-His/lacZ. Cells were lysed $48 \mathrm{~h}$ after transfection (with Reporter Lysis Buffer, Roche Diagnostics), and luciferase activity was measured with the Luciferase Assay System (Promega) and normalized to $\beta$-galactosidase activity, which was measured by means of the Galacto-Light Plus System (Applied Biosystems).

Case selection. Primary invasive breast carcinomas from 103 postmenopausal women (range 50-89 years) were retrieved from the files of the Department of Pathology of the University Hospital of the Katholieke Universiteit Leuven, Belgium. All patients underwent mastectomy or local wide excision with axillary lymph node dissection between 2002 and 2006 . For each case, both snap-frozen and formalin-fixed paraffin-embedded tumor tissue blocks were available. Breast carcinomas were typed and graded according to the WHO Classification and the Elston and Ellis (13) grading system, respectively.

Quantitative real-time PCR. For quantitative real-time PCR (RT-PCR) analysis, we used frozen tissue blocks that had been stored at $-80^{\circ} \mathrm{C}$. Breast cancer cases consisted of at least $50 \%$ invasive tumor cells. As a control, we included snap-frozen biopsy specimens representing normal premenopausal $(n=5)$ and postmenopausal $(n=14)$ breast tissue obtained from mammoplastic surgery specimens. Total RNA was extracted from eight to ten 20- $\mu \mathrm{m}$ sections of each frozen tissue block, using the RNeasy kit (Qiagen). One microgram RNA was reverse transcribed and PCR reactions on the resulting cDNA were done in the ABI-prism 7700 sequence detector (Applied Biosystems). PCR primers and fluorogenic probes (FAM or VIC as reporter dyes and TAMRA or BHQ as quencher dyes) for mouse $\beta$-actin, mouse Chk1, mouse Claspin, human Chk1, human Claspin, human E2Fl, human $\mathrm{Ki}-67$, and human $18 \mathrm{~S}$ RNA were purchased from Eurogentec. Sequences of primers and probes are available upon request. Expression of $\beta$-actin (for MC3T3-E1 cells) or 18S RNA (for breast tissue) was used to normalize gene expression.

Immunohistochemistry. All breast cancer samples were examined for the expression of ER (clone SP1, dilution 1/100, Labvision Corporation), PR (clone SP2, dilution 1/500, Labvision Corporation), and HER-2 (clone CB11, dilution $1 / 40$, Novocastra Laboratories). For ER and PR, any nuclear staining of invasive tumor cells was considered as positive. HER-2 immunostaining was scored according to the guidelines for HercepTest (14) and a positive HER-2 status was confirmed by fluorescence in situ hybridization (PathVision, Vysis).
A

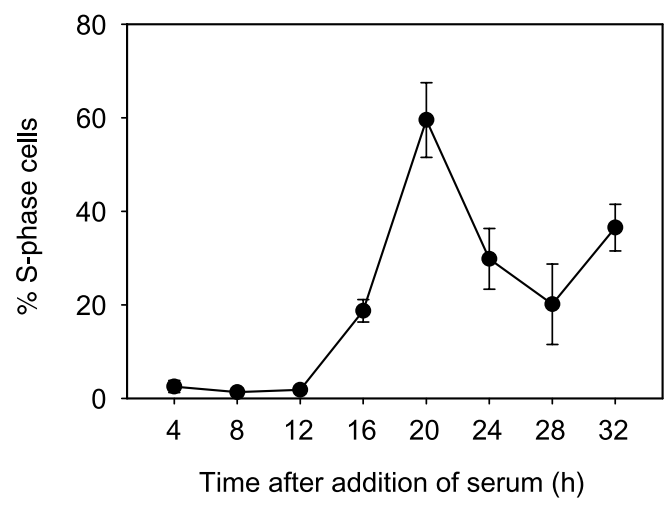

B

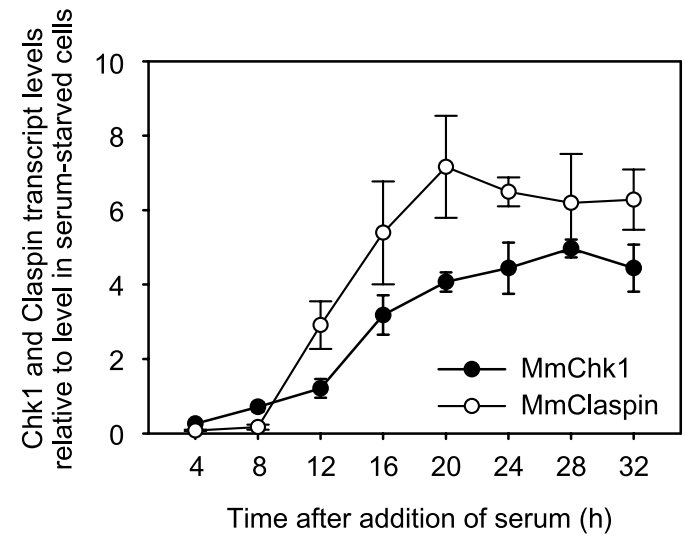

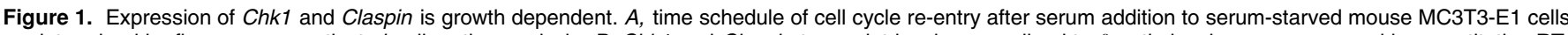

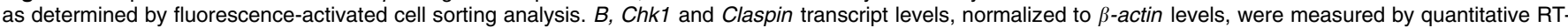
PCR after addition of serum to MC3T3-E1 cells that were synchronized in the $\mathrm{G}_{1}$ phase of the cell cycle by serum starvation. Points, mean of three independent experiments; bars, SE. 


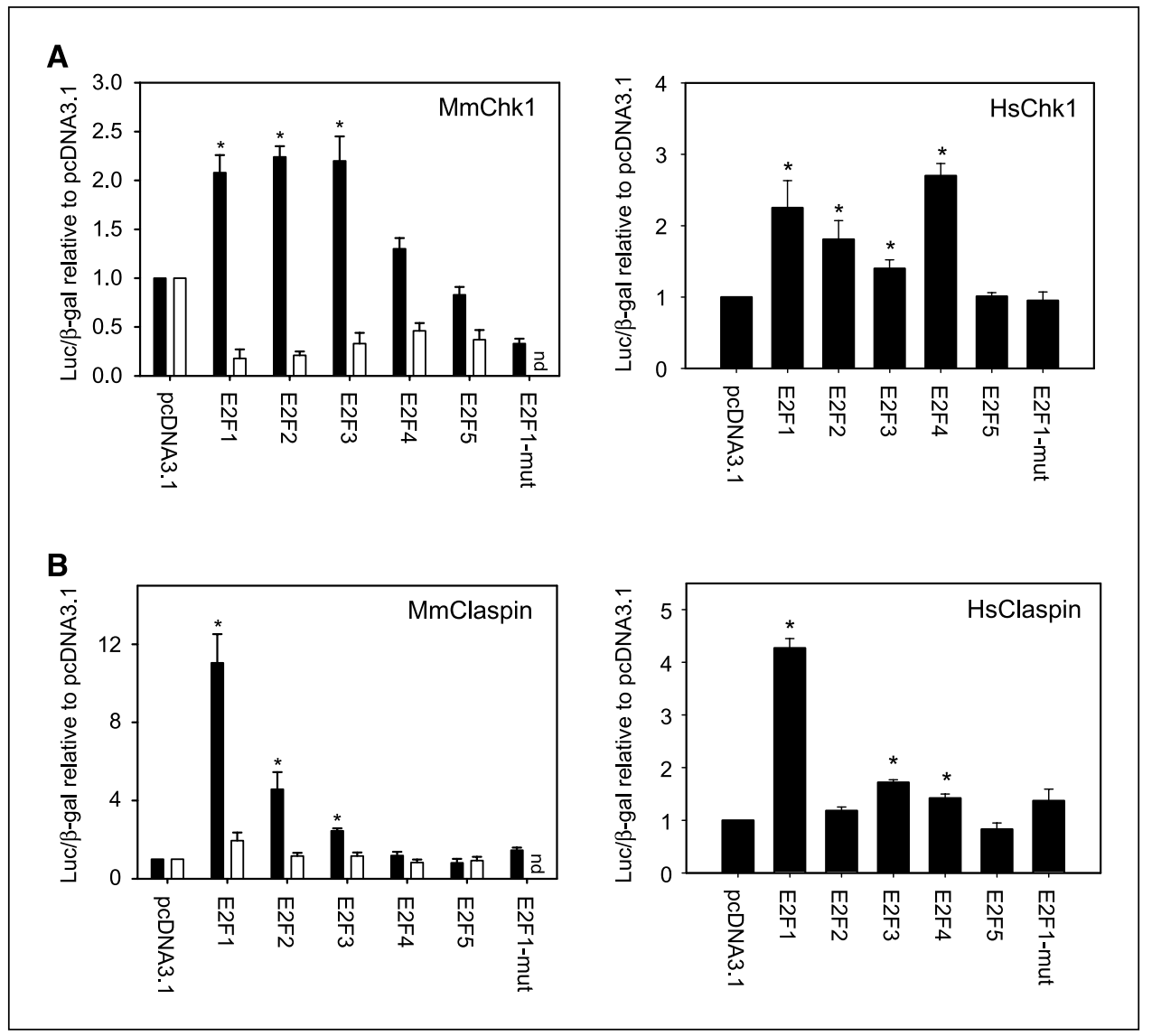

Figure 2. Promoter activity of Chk1 and Claspin is regulated by E2F transcription factors. $A$ and $B$, left, exponentially growing MC3T3-E1 cells were transfected with an E2F expression vector, the corresponding empty control expression vector, or an expression vector for mutant E2F1, with a $\beta$-galactosidase expression vector and with a luciferase reporter construct that carried either a mouse Chk 1 $(A)$ or Claspin $(B)$ promoter fragment that contained an intact (black columns) or mutated (white columns) E2F-responsive site. $A$ and $B$, right, exponentially growing MCF-7 cells were transfected with an E2F expression vector, the corresponding empty control expression vector, or an expression vector for mutant E2F1, with a $\beta$-galactosidase expression vector and with a luciferase reporter construct that carried either a human $\operatorname{Chk1}(A)$ or Claspin $(B)$ promoter fragment that contained the E2F-responsive site. In all transfection experiments, cells were lysed $48 \mathrm{~h}$ after transfection. Luciferase activities, normalized for $\beta$-galactosidase activities, of the different reporter constructs were standardized against the empty $p G L 3$ vector. Furthermore, induction by the different E2F expression plasmids was expressed relative to the pcDNA3.1. Columns, mean of at least three independent experiments; bars, SE. *, $P<0.05$, E2F overexpression versus empty vector control pcDNA3.1 (Student's $t$ test)

Protein expression of Chk1, E2F1, and Ki67, for which commercial antibodies were available, was determined in a selected series including three grade 1, three grade 2, and nine grade 3 tumors. For E2F1 (Clone KH95, 1/100 diluted, Santa Cruz Biotechnology, Inc.) and Ki-67 (clone Mib-1, 1/100 diluted, DakoCytomation), the percentage of positively stained tumor nuclei was scored as follows: $0 \%$, negative; $1 \%$ to $10 \%, 1+; 11 \%$ to $25 \%, 2+$; $26 \%$ to $50 \%, 3+$, and $>50 \%$, 4+. For Chk1 (clone EP691Y, 1/400 diluted, Epitomics), the intensity of nuclear and/or cytoplasmic staining of invasive tumor cells was scored on a 0 to $4+$ scale.

Breast carcinomas with a triple-negative ER-/PR-/HER-2- status were additionally stained for high-molecular-weight cytokeratins CK5.6 (clone D516B4, dilution 1/50, DakoCytomation), CK14 (clone LL002, 1/10 diluted, Novocastra Laboratories), and CK17 (clone E3, 1/20 diluted, DakoCytomation) and for epidermal growth factor receptor (EGFR; PharmDx kit, prediluted, DakoCytomation). For CK5/6, CK14, and CK17, a threshold of $\geq 10 \%$ of invasive tumor cells showing cytosolic staining was adopted (15). EGFR immunostaining was scored according to the guidelines for HercepTest (14). Triple-negative tumors positive for at least one highmolecular-weight cytokeratin and/or EGFR were considered to have a basal-like phenotype (16).

Statistics. All statistical analyses were done with the software program Statistica (StatSoft, Inc.). Results from in vitro experiments in MC3T3-E1 and MCF-7 cells were expressed as the means and SE of at least three independent experiments. Significance of the overall up-regulation of gene expression after addition of serum to serum-starved cells was assessed by the Fisher's least significant difference multiple comparison test. For the transfection experiments, differences between E2F overexpression and empty vector control (pcDNA3.1) were analyzed by Student's $t$ test. Differences in gene expression levels across patient groups, defined by histologic tumor grade; lymph node metastases; ER, PR, or HER-2 status; and tumor size were assessed by the Mann-Whitney $U$ (two groups) or Kruskal-Wallis (three or more groups) tests. The Pearson product-moment correlation coefficient was used to evaluate the associations between $C h k l$, Claspin, E2F1, and Ki-67. Logistic regression was implemented to determine whether Chk1, Claspin, E2F1, or Ki-67 expression was a significant predictor of histologic tumor grade; lymph node metastasis; or ER, PR, or HER-2 status. Considering grade 3 breast carcinomas separately, we further examined whether Chkl, Claspin, E2F1, or Ki-67 gene expression levels could predict ER, PR, and/or HER-2 status.

\section{Results}

Expression of Chkl and Claspin is growth-related and regulated by E2F transcription factors. To determine whether the expression of Chkl and Claspin was growth-regulated, mouse MC3T3-E1 cells were serum starved for $48 \mathrm{~h}$ and subsequently stimulated to re-enter the cell cycle by addition of serum (Fig. 1A). As shown in Fig. $1 B$, mRNA levels of Chkl and Claspin increased after the addition of serum, suggesting that the expression of these genes was growth regulated. Promoter constructs of mouse Chk1 and Claspin, which carry E2Fresponsive sites, were transfected in MC3T3-E1 cells together with E2F expression plasmids to investigate whether exogenous expression of E2F transcription factors could enhance Chk1 and Claspin promoter activities. E2F1, E2F2, and E2F3 were able to transactivate reporter constructs that were driven by the promoter regions of Chk1 (Fig. 2A, left, black columns; 2.1-2.2fold induction) or Claspin (Fig. 2B, left, black columns; 2.5- to 11.1-fold induction). However, mutant E2F1 was unable to transactivate the mouse Chkl or Claspin promoter construct. Mutation of the E2F-responsive elements within these promoter constructs also completely abolished their responsiveness to E2F 
(Fig. $2 A$ and $B$, left, white columns). The sequence of the E2Fbinding sites was well conserved between mouse and human. Human promoter constructs were transfected in human MCF-7 breast cancer cells together with different E2F family members. $\mathrm{E} 2 \mathrm{~F} 1, \mathrm{E} 2 \mathrm{~F} 2, \mathrm{E} 2 \mathrm{~F} 3$, and $\mathrm{E} 2 \mathrm{~F} 4$ were able to transactivate reporter constructs that were driven by the promoter regions of human Chk1 (Fig. 2A, right; 1.4- to 2.3-fold induction) or Claspin (Fig. 2B, right; 1.4- to 4.3-fold induction).

Transcript levels of ChkI and Claspin are highly correlated with $E 2 F 1$ transcript levels in human breast carcinomas. As shown in Fig. 3, expression levels of Chk1, Claspin, E2F1, and Ki-67 were consistently low in normal postmenopausal breast tissue as opposed to premenopausal tissue. In postmenopausal breast carcinomas, there was a strong and statistically significant correlation between $C h k 1$ and E2F1 expression (Pearson productmoment correlation coefficient of 0.66) as well as between Claspin and E2F1 expression (correlation coefficient of 0.74). Transcript levels of $C h k 1$ and Claspin also correlated very well (correlation coefficient of 0.84). Moreover, expression levels of Chkl, Claspin,

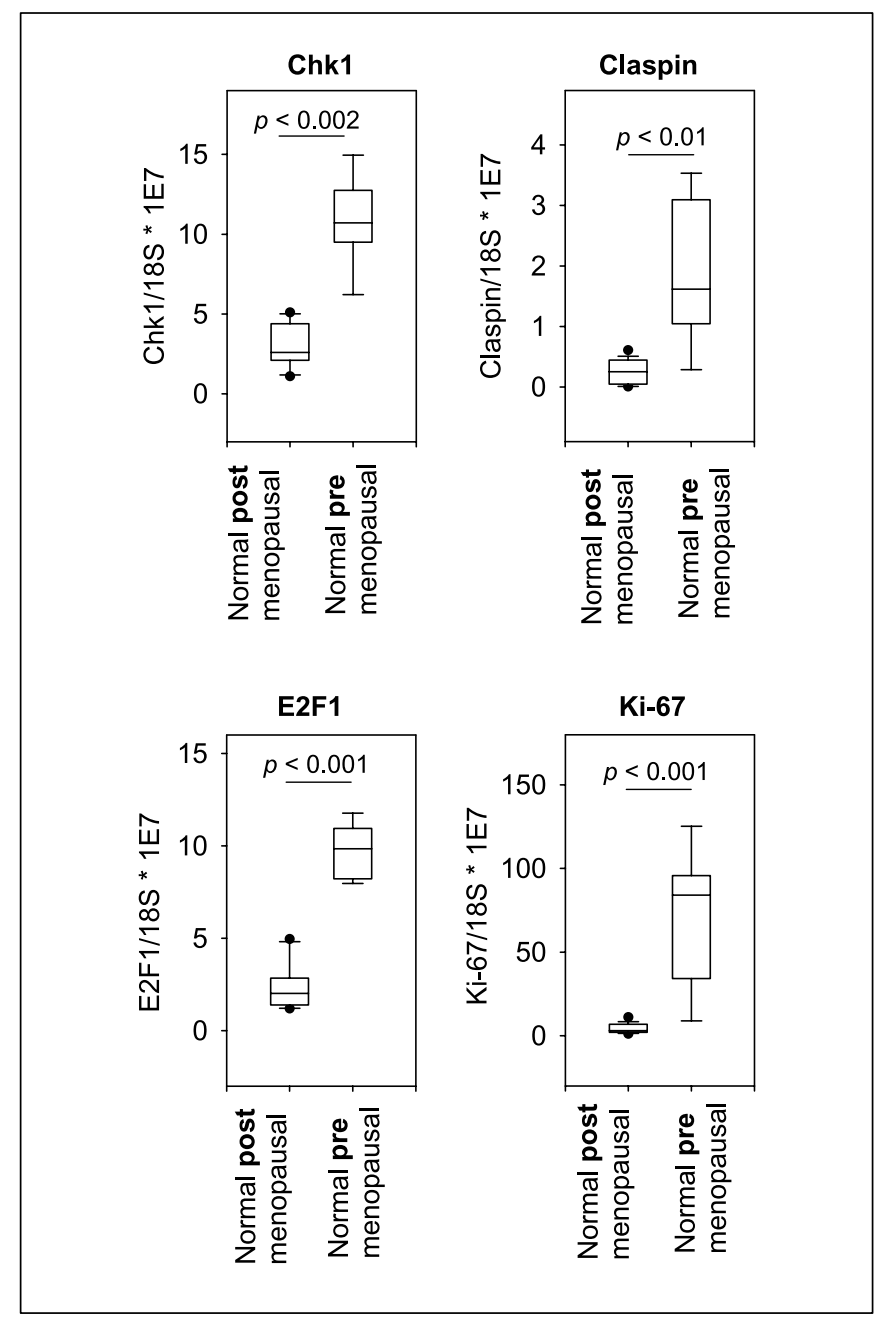

Figure 3. Chk1 and Claspin are more abundantly expressed in normal premenopausal breast tissue. Transcript levels of Chk1, Claspin, E2F1, and Ki-67 were determined in normal premenopausal $(n=5)$ and postmenopausal ( $n=14$ ) breast tissue obtained from mammoplastic surgery specimens. Mann-Whitney $U$ tests were applied to investigate expression differences between premenopausal and postmenopausal breast tissue. and E2F1 correlated strongly with the expression level of the proliferation marker $\mathrm{Ki}-67$ (correlation coefficients of $0.73,0.78$, and, 0.57 , respectively).

Evaluation of Chk1, Claspin, E2F1, and Ki-67 transcript levels in postmenopausal breast carcinomas. Gene expression levels of Chk1, Claspin, E2F1, and Ki-67 were significantly higher in tumors showing large tumor size $(>2 \mathrm{~cm})$, high histologic grade, and lack of ER and/or PR expression (Table 1). No significant expression differences were found between HER-2-positive and HER-2-negative tumors. Neither was there a correlation with the presence of lymph node metastases at diagnosis.

Transcript levels of the four investigated genes were higher in triple-negative ER-/PR-/HER-2- grade 3 breast carcinomas compared with all other grade 3 tumors (Table 2). When corrected for multiple comparisons, significance was only reached for $C h k 1$ $(P<0.0001)$ and $K i-67(P<0.01)$. In logistic regression analysis, $C h k 1$ was found to be the most powerful single predictor of the triple-negative status of breast tumors (area under receiver operating characteristic curve of $0.83 ; P<0.01$ ).

Immunohistochemistry. Protein expression of E2F1, Ki-67, and Chkl was generally higher in high-grade compared with low-grade carcinomas (Fig. 4). In particular, triple-negative ER-/PR-/HER2 - breast carcinomas tended to show stronger Chk1 expression compared with other grade 3 tumors.

Twelve of $16(75 \%)$ triple-negative tumors showed a basal-like immunophenotype (Supplementary Table). Additional staining for "basal-like markers" in triple-negative breast cancer was not correlated with higher expression levels of Chkl, Claspin, E2F1, or Ki-67.

\section{Discussion}

In the present study, we investigated the transcriptional regulation of Chk1 and Claspin by the E2F family of transcription factors and examined $C h k 1$ and Claspin transcript levels in a set of 103 primary invasive breast carcinomas. A first indication of the E2F dependency of the Chk1 and Claspin promoter activity was obtained by the observation that Chkl and Claspin transcript levels were growth-regulated both in vitro and in vivo. Indeed, Chkl and Claspin mRNA expression levels were significantly down-regulated in the serum-arrested mouse MC3T3-E1 cells. Likewise, Chk1 and Claspin transcript levels were significantly lower in normal postmenopausal breast tissue in comparison with normal premenopausal breast tissue, which is known to contain a larger proportion of hormone dependent, proliferating cells (16). Transient transfection experiments illustrated that both mouse and human Chkl and Claspin promoter activities were induced upon cotransfection of activating E2F family members. However, further studies are required to determine the contribution of posttranscriptional stabilization to the transactivation of $C h k 1$ and Claspin by the E2F transcription factors in vivo.

In our series of primary invasive breast carcinomas, we found a strong correlation between the expression levels of Chkl and Claspin. This correlation is in agreement with the finding that Chkl overexpression increases Claspin levels (17). Furthermore, we found a tight correlation between the expression levels of both Chk1 and Claspin and the proliferation marker Ki-67. These findings are consistent with the data of Tort et al. (18), that human lymphomas with high proliferative activity showed higher levels of Chk1 mRNA and protein and confirm recent data of Tsimaratou et al. (19), who suggested the use of Claspin as a proliferation 


\begin{tabular}{|c|c|c|c|c|c|c|c|c|c|}
\hline \multirow[t]{2}{*}{ Characteristics } & \multirow[t]{2}{*}{ Cases, $n(\%)$} & \multicolumn{2}{|c|}{ Chk1* } & \multicolumn{2}{|c|}{ Claspin $^{\dagger}$} & \multicolumn{2}{|c|}{$E 2 F 1^{\ddagger}$} & \multicolumn{2}{|c|}{$K i-67^{\S}$} \\
\hline & & Mean \pm SE & $P^{\|}$ & Mean \pm SE & $P^{\|}$ & Mean \pm SE & $P^{\|}$ & Mean \pm SE & $P^{\|}$ \\
\hline \multicolumn{10}{|l|}{ Menopausal status } \\
\hline Premenopausal & 0 & & & & & & & & \\
\hline Postmenopausal & $103(100.0)$ & $10.9 \pm 1.1$ & & $4.6 \pm 0.5$ & & $40.2 \pm 5.3$ & & $94.7 \pm 9.0$ & \\
\hline Tumor grade & & & $<0.0001$ & & $<0.0001$ & & $<0.0001$ & & $<0.0001$ \\
\hline Grade 1 (7 ITC and 13 IDC) & $20(19.4)$ & $5.0 \pm 1.1$ & & $1.3 \pm 0.2$ & & $11.8 \pm 2.1$ & & $30.4 \pm 4.2$ & \\
\hline Grade 2 (11 ILC and 10 IDC) & $21(20.4)$ & $4.2 \pm 0.5$ & & $1.6 \pm 0.2$ & & $16.0 \pm 2.4$ & & $33.0 \pm 4.5$ & \\
\hline Grade 3 (10 ILC and 52 IDC) & $62(60.2)$ & $15.1 \pm 1.6$ & & $6.6 \pm 0.8$ & & $57.6 \pm 8.0$ & & $138.0 \pm 12.3$ & \\
\hline Tumor size $(\mathrm{cm})$ & & & 0.0336 & & 0.0006 & & 0.0022 & & 0.0045 \\
\hline$\leq 2$ & $63(61.2)$ & $8.6 \pm 1.6$ & & $3.2 \pm 0.8$ & & $31.6 \pm 10.5$ & & $71.5 \pm 12.3$ & \\
\hline$>2$ & $40(38.8)$ & $12.4 \pm 1.5$ & & $5.5 \pm 0.7$ & & $45.7 \pm 5.6$ & & $110.9 \pm 12.3$ & \\
\hline Lymph node status & & & 0.2548 & & 0.8495 & & 0.5531 & & 0.8125 \\
\hline Negative & $42(40.8)$ & $10.4 \pm 1.2$ & & $4.5 \pm 0.5$ & & $37.6 \pm 5.5$ & & $97.2 \pm 12.7$ & \\
\hline Positive & $61(59.2)$ & $11.7 \pm 2.0$ & & $4.7 \pm 1.1$ & & $43.9 \pm 10.4$ & & $93.2 \pm 12.7$ & \\
\hline ER status & & & $<0.0001$ & & $<0.0001$ & & $<0.0001$ & & $<0.0001$ \\
\hline Negative & $31(30.1)$ & $18.9 \pm 2.2$ & & $7.6 \pm 1.1$ & & $65.4 \pm 13.2$ & & $162.7 \pm 20.3$ & \\
\hline Positive & $72(69.9)$ & $7.5 \pm 1.0$ & & $3.2 \pm 0.5$ & & $29.3 \pm 4.6$ & & $66.7 \pm 7.5$ & \\
\hline PR status & & & $<0.0001$ & & $<0.0001$ & & $<0.0001$ & & $<0.0001$ \\
\hline Negative & $40(38.8)$ & $17.0 \pm 2.0$ & & $6.9 \pm 1.0$ & & $57.6 \pm 10.7$ & & $146.5 \pm 17.5$ & \\
\hline Positive & $63(61.2)$ & $7.1 \pm 1.0$ & & $3.1 \pm 0.7$ & & $29.2 \pm 5.0$ & & $63.3 \pm 7.5$ & \\
\hline HER-2 status & & & 0.3957 & & 0.0093 & & 0.0352 & & 0.0284 \\
\hline Negative & 73 (70.9) & $11.5 \pm 1.5$ & & $4.4 \pm 0.7$ & & $39.1 \pm 7.1$ & & $92.2 \pm 12.2$ & \\
\hline Positive & $30(29.1)$ & $9.5 \pm 1.0$ & & $4.9 \pm 0.5$ & & $42.8 \pm 5.8$ & & $103.8 \pm 9.9$ & \\
\hline
\end{tabular}

Abbreviations: ITC, invasive tubular carcinoma; IDC, invasive ductal carcinoma-no specific type; ILC, invasive lobular carcinoma.

${ }^{*}$ Chk1 $18 S^{*} 1 \mathrm{E} 7$.

†Claspin $/ 18 S^{*} 1 \mathrm{E} 7$.

$\ddagger E 2 F 1 / 18 S^{*} 1 \mathrm{E} 7$.

$\S \mathrm{Ki}-67 / 18 S^{*} 1 \mathrm{E} 7$.

\|Unadjusted $P$ values according to Mann-Whitney $U$ test (two groups) or Kruskal-Wallis test (three groups).

marker in human cancer and normal tissues. Interestingly, we found concurrent up-regulation of all three markers, Ki-67, Chkl, and Claspin, with E2F1. Whereas coregulation of Ki-67 and E2F1 has been shown before $(20,21)$, our data strongly support the findings of microarray studies, showing that proliferation-associated genes are often concurrently up-regulated in breast cancer, which is called a proliferation cluster (22). Many of these proliferationassociated genes are shown to contain binding sites for the E2F transcription factors and $E 2 F 1$ itself is a member of this proliferation signature (23).

\begin{tabular}{|c|c|c|c|c|c|c|c|c|c|}
\hline \multirow[t]{2}{*}{ Characteristics } & \multirow{2}{*}{$\begin{array}{c}\text { Cases, } \\
n(\%)\end{array}$} & \multicolumn{2}{|c|}{ Chk $1^{*}$} & \multicolumn{2}{|c|}{ Claspin $^{\dagger}$} & \multicolumn{2}{|c|}{$E 2 F 1^{\ddagger}$} & \multicolumn{2}{|c|}{$K i-67^{\S}$} \\
\hline & & Mean \pm SE & $P^{\|}$ & Mean \pm SE & $P^{\|}$ & Mean \pm SE & $P^{\|}$ & Mean \pm SE & $P^{\|}$ \\
\hline Tumor type & & & $<0.0001$ & & 0.1221 & & 0.2244 & & 0.0020 \\
\hline $\begin{array}{l}\text { ER-, PR-, } \\
\text { HER-2-negative tumors }\end{array}$ & $16(26.2)$ & $25.8 \pm 3.3$ & & $9.1 \pm 2.0$ & & $74.3 \pm 24.0$ & & $206.2 \pm 33.7$ & \\
\hline Other grade 3 tumors & $45(73.8)$ & $11.4 \pm 1.7$ & & $5.7 \pm 0.8$ & & $51.9 \pm 7.2$ & & $114.4 \pm 10.1$ & \\
\hline
\end{tabular}

${ }^{*}$ Chk1 $18 S^{*} 1 \mathrm{E} 7$

†Claspin/18S*1E7.

$\ddagger E 2 F 1 / 18 S^{*} 1 \mathrm{E} 7$.

$\S \mathrm{Ki}-67 / 18 S^{*} 1 \mathrm{E} 7$.

\|Unadjusted $P$ values according to Mann-Whitney $U$ test (two groups) or Kruskal-Wallis test (three groups). 
The expression of $K i-67$, and also of Chkl, Claspin, and E2F1, was strongly associated with histologic tumor grade and lack of ER and/or PR expression, but not with HER-2 status and lymph node status. Immunohistochemical stainings illustrated that also at the protein level, the highest expression of Chk1, E2F1, and Ki-67 was found in grade 3 tumors. Most of these findings on Ki-67 are in agreement with previous reports (24). Interestingly, we found that the transcript levels of $K i-67$ and especially $C h k 1$ were significantly higher in grade 3 breast carcinomas showing a triple-negative ER-/PR-/HER-2- status compared with other grade 3 tumors. In parallel, protein levels of Chkl and Ki-67 tended to be increased in grade 3 triple-negative breast carcinomas. These data fit with the intrinsic breast cancer subtypes revealed by gene expression profiling studies. These have identified at least four distinct breast cancer subgroups that have prognostic as well as predictive implications (25-27): ER-positive or luminal, ER-negative/HER-2positive, normal breast-like, and basal-like tumors. The latter basallike tumors show an aggressive clinical behavior. They are characterized by a triple-negative ER-/PR-/HER-2-status, the expression of high-molecular-weight cytokeratins, and overexpression of proliferation-associated genes and/or EGFR (26, 28, 29). Further studies have shown that these tumors encompass as many as $25 \%$ of grade 3 breast carcinomas and that they are associated with a high mitotic index $(30,31)$. Most of the triple-negative tumors in our study showed a basal-like immunophenotype (12 of $16,75 \%)$ as they expressed one or more high-molecular-weight cytokeratins and/or EGFR, which is in line with the literature (32). However, additional staining for basal-like markers in triplenegative breast cancer was not correlated with higher expression levels of Chk1, Claspin, E2F1, or Ki-67.

Whereas ER- and/or PR-positive breast carcinomas can be treated with antiestrogen therapy and HER-2-positive carcinomas with anti-HER-2 monoclonal antibody therapy, no targeted therapeutics are available for triple-negative ER-/PR-/HER-2tumors, including the basal-like subtype. At present, chemotherapy is the only option for the latter and success rates have been controversial $(33,34)$. Therefore, novel therapeutic approaches are mandatory for the triple-negative breast cancer patient. In this context, inhibition of EGFR signaling by either anti-EGFR monoclonal antibodies or small-molecule tyrosine kinase inhibitors has been proposed because EGFR overexpression has been reported in a substantial proportion of triple-negative and/or basal-like breast carcinomas $(35,36)$. In our series of 16 triplenegative tumors, six cases showed EGFR overexpression (37.5\%).
Figure 4. Immunohistochemical staining of Chk1, E2F1, and Ki-67 in breast carcinoma. Invasive lobular carcinoma, classic-type (grade 2; $A-C$ ) compared with grade 3 invasive ductal carcinoma, triple ER-/PR-/HER-2- $(D-F)$. A, weak cytoplasmic/nuclear Chk1 staining (score $1+$ ) compared with $(D)$ strong, mainly cytoplasmic Chk1 staining (score 4+). B, nuclear E2F1 staining in $<10 \%$ of invasive tumor cells (score $1+$ ) compared with $(E)$ nuclear E2F1 staining in at least $50 \%$ of invasive tumor cells (score 4+). $C$, nuclear Ki-67 staining in $<10 \%$ of invasive tumor cells (score $1+$ ) compared with $(F)$ nuclear Ki-67 staining in at least $50 \%$ of invasive tumor cells (score 4+). Original magnification, $\times 400$.

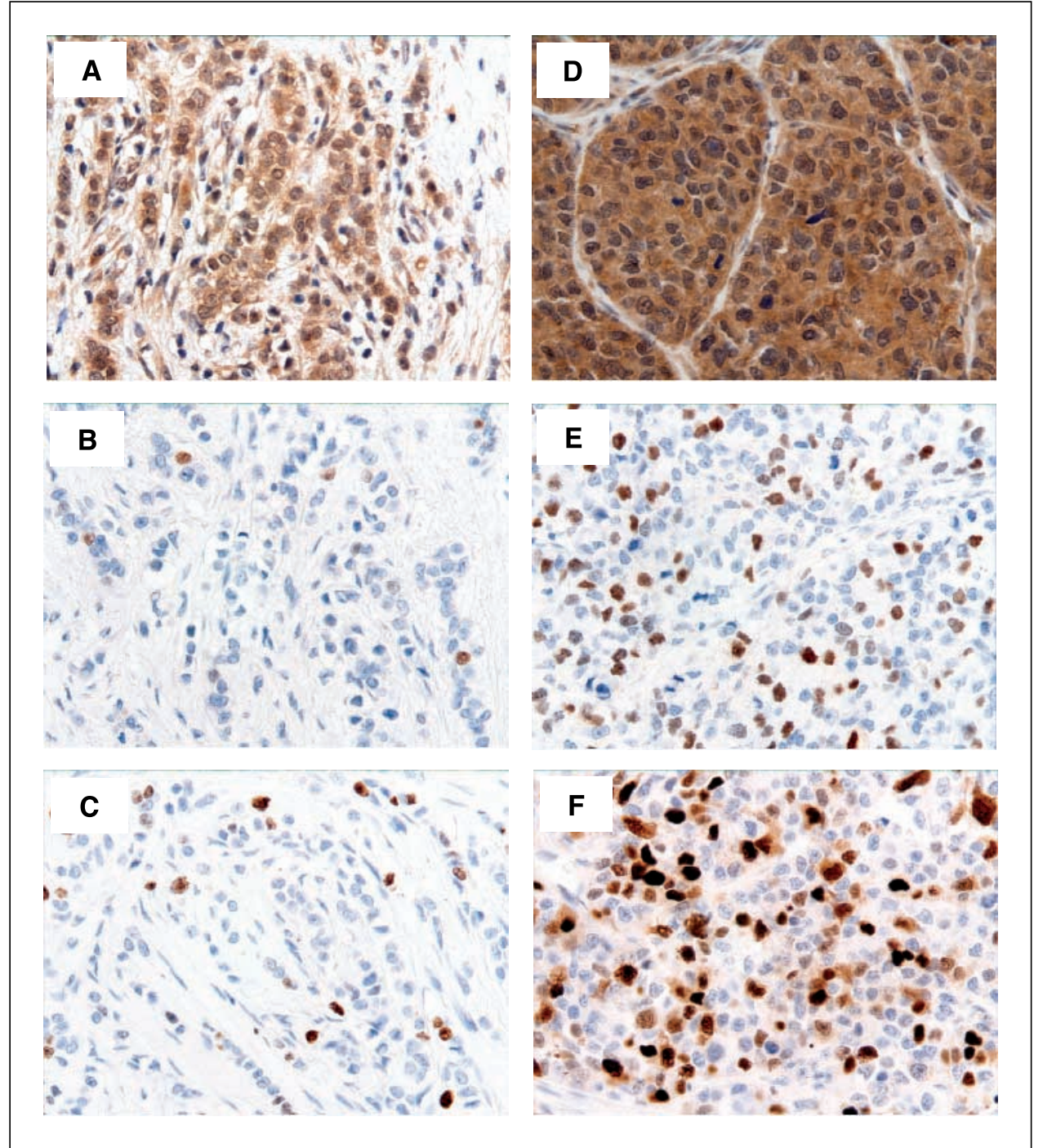


Unfortunately, targeted anti-EGFR therapy in breast cancer has been quite disappointing thus far and in contrast to anti-HER-2targeting, it is as yet unclear which patients are likely to benefit from anti-EGFR therapy $(37,38)$. In the present study, we found higher expression of the checkpoint proteins Claspin and especially Chk1 in triple-negative breast carcinomas. Interestingly, it has been shown that inhibition of checkpoint signaling enhances the effect of concomitant treatment with DNA-damaging drugs or radiation. Especially, targeting of the kinase Chkl was suggested to be effective in the treatment of cancer (39). Different Chk1-inhibitors, among which 7-hydroxystaurosporine (UCN-01), the indocarbazole Go6976, isogranulatimide, CEP-3891, and CHIR-124, have been shown both in vitro as in vivo to increase chemosensitivity and radiosensitivity in tumor cells (ref. 40 and references herein; refs. 41, 42). Triple-negative breast carcinomas frequently harbor alterations in DNA damage response pathways such as p53 and BRCA1, both of which are critically involved in the $\mathrm{G}_{1}-\mathrm{S}$ phase checkpoint; as a result, these tumor cells largely depend on the $\mathrm{G}_{2^{-}}$ M checkpoint in case of DNA damage $(31,43,44)$. It would therefore be interesting to investigate whether anti-Chk1-containing therapeutic regimens, which sensitize to DNA damage, would be useful in the treatment of triple-negative breast carcinomas. A major challenge in this field would concern specific targeting of tumor cells because Chk1 is essential for the maintenance of genomic integrity also in normal cells. To this end, a new generation of oncolytic adenoviral vectors, expressing antisense Chkl cDNA preferentially in tumor cells, has been used in a preclinical setting and yielded promising results $(6,23)$. Alternatively, Chen et al. (45) have recently developed two potent and selective Chk1 inhibitors, A-690002 and A-641397, which potentiate the cytotoxicity of DNA-damaging agents in p53-deficient cancer cells but not in p53-proficient normal cells.

In conclusion, expression of the E2F-regulated genes $C h k 1$ and Claspin correlated strongly with several adverse clinicopathologic variables in breast cancer. Moreover, Chkl expression was significantly higher in grade 3 breast carcinomas showing a triple-negative ER-/PR-/HER-2- phenotype compared with other grade 3 tumors. Further research is warranted to validate the use of Chkl inhibitors in triple-negative breast carcinomas for which treatment strategies are limited at present.

\section{Acknowledgments}

Received 9/25/2006; revised 5/3/2007; accepted 5/11/2007.

Grant support: Fund for Scientific Research (FWO-G.0508.05 and FWO-G.0553.06) and the KU Leuven Research Council (EF/05/007 SymBioSys).G. Eelen is a postdoctoral fellow of the Fund for Scientific Research.

The costs of publication of this article were defrayed in part by the payment of page charges. This article must therefore be hereby marked advertisement in accordance with 18 U.S.C. Section 1734 solely to indicate this fact.

We thank I. Beullens, S. Marcelis, L. Ophalvens, B.K. Tan, M. Van Camp, C. Van Den Broeck, and E. Vanoirbeek for excellent technical assistance; and Drs. J.R. Nevins, J. Magae, and K. Helin for providing expression plasmids.

\section{References}

1. Bartek J, Lukas J. Chk1 and Chk2 kinases in checkpoint control and cancer. Cancer Cell 2003;3:421-9.

2. Kastan MB, Bartek J. Cell-cycle checkpoints and cancer. Nature 2004;432:316-23.

3. Kumagai A, Kim SM, Dunphy WG. Claspin and the activated form of ATR-ATRIP collaborate in the activation of Chkl. J Biol Chem 2004;279:49599-608.

4. Sar F, Lindsey-Boltz LA, Subramanian D, et al. Human claspin is a ring-shaped DNA-binding protein with high affinity to branched DNA structures. J Biol Chem 2004, 279:39289-95.

5. Lin SY, Li K, Stewart GS, Elledge SJ. Human Claspin works with BRCAl to both positively and negatively regulate cell proliferation. Proc Natl Acad Sci U S A 2004, 101:6484-9.

6. Gao Q, Zhou J, Huang X, et al. Selective targeting of checkpoint kinase 1 in tumor cells with a novel poten oncolytic adenovirus. Mol Ther 2006;13:928-37.

7. Graves PR, Yu L, Schwarz JK, et al. The Chk1 protein kinase and the $\mathrm{Cdc} 25 \mathrm{C}$ regulatory pathways are targets of the anticancer agent UCN-01. J Biol Chem 2000;275: 5600-5.

8. Tao ZF, Lin NH. Chkl inhibitors for novel cance treatment. Anticancer Agents Med Chem 2006;6: 377-88.

9. Clarke CA, Bennett LN, Clarke PR. Cleavage of claspin by caspase-7 during apoptosis inhibits the Chkl pathway. J Biol Chem 2005;280:35337-45.

10. Verlinden L, Eelen G, Van Hellemont R, et al. $1 \alpha, 25$ Dihydroxyvitamin D(3)-induced down-regulation of the checkpoint proteins, Chkl and Claspin, is mediated by the pocket proteins p107 and p130. J Steroid Biochem Mol Biol 2007;103:411-5.

11. Iwanaga $R$, Komori $H$, Ishida $S$, et al. Identification of novel E2F1 target genes regulated in cell cycledependent and independent manners. Oncogene 2006; 25:1786-98.

12. Carrassa L, Broggini M, Vikhanskaya F, Damia G. Characterization of the 5 flanking region of the human Chkl gene: identification of E2F1 functional sites. Cell Cycle 2003;2:604-9.
13. Elston EW, Ellis IO. Method for grading breast cancer. J Clin Pathol 1993;46:189-90.

14. Jacobs TW, Gown AM, Yaziji H, Barnes MJ, Schnitt S . Specificity of HercepTest in determining HER-2/neu status of breast cancers using the United States Food and Drug Administration-approved scoring system. J Clin Oncol 1999;17:1983-7.

15. Reis-Filho JS, Milanezi F, Steele D, et al. Metaplastic breast carcinomas are basal-like tumours. Histopathology 2006;49:10-21.

16. Anderson TJ, Ferguson DJ, Raab GM. Cell turnover in the "resting" human breast: influence of parity, contraceptive pill, age and laterality. Br J Cancer 1982;46:376-82. 17. Chini CC, Wood J, Chen J. Chkl is required to maintain claspin stability. Oncogene 2006;25:4165-71.

18. Tort F, Hernandez S, Bea S, et al. Checkpoint kinase 1 (CHKl) protein and mRNA expression is downregulated in aggressive variants of human lymphoid neoplasms. Leukemia 2005;19:112-7.

19. Tsimaratou K, Kletsas D, Kastrinakis NG, et al. Evaluation of claspin as a proliferation marker in human cancer and normal tissues. J Pathol 2007;211:331-9.

20. Saiz AD, Olvera M, Rezk S, Florentine BA, McCourty A, Brynes RK. Immunohistochemical expression of cyclin D1, E2F-1, and Ki-67 in benign and malignant thyroid lesions. J Pathol 2002;198:157-62.

21. Zhang SY, Liu SC, Al Saleem LF, et al. E2F-1: a proliferative marker of breast neoplasia. Cancer Epidemiol Biomarkers Prev 2000;9:395-401.

22. Sorlie T. Molecular portraits of breast cancer: tumour subtypes as distinct disease entities. Eur Cancer 2004;40:2667-75.

23. Whitfield ML, George LK, Grant GD, Perou CM. Common markers of proliferation. Nat Rev Cancer 2006; 6:99-106.

24. Trihia H, Murray S, Price K, et al. Ki-67 expression in breast carcinoma: its association with grading systems, clinical parameters, and other prognostic factors-a surrogate marker? Cancer 2003;97:1321-31.

25. Sotiriou C, Neo SY, McShane LM, et al. Breast cancer classification and prognosis based on gene expression profiles from a population-based study. Proc Natl Acad Sci U S A 2003;100:10393-8.
26. Perou CM, Sorlie $\mathrm{T}$, Eisen $\mathrm{MB}$, et al. Molecular portraits of human breast tumours. Nature 2000;406: 747-52.

27. Abd El-Rehim DM, Ball G, Pinder SE, et al. Highthroughput protein expression analysis using tissue microarray technology of a large well-characterised series identifies biologically distinct classes of breast cancer confirming recent cDNA expression analyses. Int J Cancer 2005;116:340-50.

28. Sorlie T, Perou CM, Tibshirani $R$, et al. Gene expression patterns of breast carcinomas distinguish tumor subclasses with clinical implications. Proc Natl Acad Sci U S A 2001;98:10869-74.

29. Tsuda H, Takarabe $T$, Hasegawa F, Fukutomi $T$, Hirohashi S. Large, central acellular zones indicating myoepithelial tumor differentiation in high-grade invasive ductal carcinomas as markers of predisposition to lung and brain metastases. Am J Surg Pathol 2000;24:197-202. 30. Van de Rijn M., Perou CM, Tibshirani R, et al Expression of cytokeratins 17 and 5 identifies a group of breast carcinomas with poor clinical outcome. Am J Pathol 2002;161:1991-6.

31. Carey LA, Perou CM, Livasy CA, et al. Race, breast cancer subtypes, and survival in the Carolina Breast Cancer Study. JAMA 2006;295:2492-502.

32. Nielsen TO, Hsu FD, Jensen K, et al. Immunohistochemical and clinical characterization of the basal-like subtype of invasive breast carcinoma. Clin Cancer Res 2004;10:5367-74.

33. Banerjee S, Reis-Filho JS, Ashley S, et al. Basal-like breast carcinomas: clinical outcome and response to chemotherapy. J Clin Pathol 2006;59:729-35.

34. Rouzier R, Perou CM, Symmans WF, et al. Breast cancer molecular subtypes respond differently to preoperative chemotherapy. Clin Cancer Res 2005;11:5678-85. 35. Bhargava R, Gerald WL, Li AR, et al. EGFR gene amplification in breast cancer: correlation with epidermal growth factor receptor mRNA and protein expression and HER-2 status and absence of EGFR-activating mutations. Mod Pathol 2005;18:1027-33.

36. Siziopikou KP, Ariga R, Proussaloglou KE, Gattuso P, Cobleigh $\mathrm{M}$. The challenging estrogen receptor-negative/ progesterone receptor-negative/HER-2-negative patient: 
a promising candidate for epidermal growth factor receptor-targeted therapy? Breast J 2006;12:360-2.

37. Green MR. Targeting targeted therapy. N Engl J Med 2004;350:2191-3.

38. Spector NL, Xia W, Burris H III, et al. Study of the biologic effects of lapatinib, a reversible inhibitor of ErbB1 and ErbB2 tyrosine kinases, on tumor growth and survival pathways in patients with advanced malignancies. J Clin Oncol 2005;23:2502-12.

39. Xiao Z, Xue J, Sowin TJ, Zhang H. Differential roles of checkpoint kinase 1 , checkpoint kinase 2, and mitogenactivated protein kinase-activated protein kinase 2 in mediating DNA damage-induced cell cycle arrest: implications for cancer therapy. Mol Cancer Ther 2006;5: 1935-43.

40. Collins I, Garrett MD. Targeting the cell division cycle in cancer: CDK and cell cycle checkpoint kinase inhibitors. Curr Opin Pharmacol 2005;5:366-73.

41. Shao RG, Cao CX, Pommier Y. Abrogation of Chk1mediated $\mathrm{S} / \mathrm{G}_{2}$ checkpoint by UCN-01 enhances ara-Cinduced cytotoxicity in human colon cancer cells. Acta Pharmacol Sin 2004;25:756-62.

42. Tse AN, Rendahl KG, Sheikh T, et al. CHIR-124, a novel potent inhibitor of Chkl, potentiates the cytotox- icity of topoisomerase I poisons in vitro and in vivo. Clin Cancer Res 2007;13:591-602.

43. Rakha EA, El Sayed $\mathrm{ME}$, Green AR, Lee $\mathrm{AH}$ Robertson JF, Ellis IO. Prognostic markers in triplenegative breast cancer. Cancer 2007;109:25-32.

44. Tischkowitz MD, Foulkes WD. The basal phenotype of BRCAl-related breast cancer: past, present and future. Cell Cycle 2006;5:963-7.

45. Chen Z, Xiao Z, Gu WZ, et al. Selective Chkl inhibitors differentially sensitize p53-deficient cancer cells to cancer therapeutics. Int J Cancer 2006;119: 2784-94. 